\title{
Silicon subwavelength waveguiding devices
}

I. Molina-Fernández ${ }^{\mathrm{a}}$, J. G. Wangüemert-Pérez ${ }^{\mathrm{a}}$, A. Ortega-Moñux ${ }^{\mathrm{a}}$, Robert Halir ${ }^{\mathrm{a}}$, J. de Oliva Rubio $^{a}$, A. Sánchez-Postigo ${ }^{a}$, J. M. Luque-González ${ }^{a}$, Abdelfettah Hadij-Elhouati ${ }^{a}$, D. PereiraMartín $^{\mathrm{a}}$, D. González-Andrade ${ }^{\mathrm{b}}$, A. V. Velasco ${ }^{\mathrm{b}}$, A. Herrero-Bermello ${ }^{\mathrm{b}}$, Jens H. Schmid ${ }^{\mathrm{c}}$, Pavel Cheben $^{\mathrm{c}}$, J. Čtyrokýd

${ }^{a}$ Universidad de Málaga, Dept. de Ingeniería de Comunicaciones, 29010 Málaga, Spain; ${ }^{\text {Innstitute of }}$ Optics, Spanish National Research Council, 28006 Madrid, Spain; ${ }^{\mathrm{C}}$ National Research Council Canada, Ottawa, Ontario K1A 0R6, Canada; ${ }^{\mathrm{d} I n s t i t u t e}$ of Photonics and Electronics, CAS, Chaberská 57, 18251 Prague, Czech Republic

Tel: (+34) 952131311, e-mail: imf@ic.uma.es

\begin{abstract}
Silicon photonics has emerged as an intense field of research due to its unique capabilities to integrate photonics and electronics into the same platform using standard semiconductor fabrication facilities. Subwavelength grating (SWG) structures, i.e. periodic nanostructured waveguides with a pitch below half the wavelength of light, allow the lossless propagation of Bloch-Floquet modes which closely resemble propagation through a homogenous waveguide with optical properties (refractive index, dispersion, birefringence) that can be tailored to fulfill specific design goals. SWG engineering is now routinely used for novel and advanced device design. Fiber-chip couplers, polarization and mode multiplexers, multimode interference couplers (MMIs), lenses, and bragg filters have been successfully designed in our group based in these concepts. In this invited talk we will review some of our last advances in the field.
\end{abstract}

Keywords: subwavelength gratings, anisotropy, homogenization, polarization management

\section{INTRODUCTION}

Diffraction is frustrated in periodic structures with a pitch below half the wavelength of light (Subwavelength Gratings, SWGs). Waveguides which are nanostructured below this limit support Floquet-Bloch modes which can propagate without losses as in a homogeneous waveguide as it was demonstrated in the seminal paper [1]. One of the advantages of SWG waveguides is that they offer additional degrees of freedom to synthesize the properties of the equivalent metamaterial and thus extends the design space available in silicon photonics. Index engineering, dispersion engineering and, more recently, birefringence engineering have been demonstrated based in this approach [2].

In the deep subwavelength regime, SWG structures can be modeled as homogeneous metamaterials whose electromagnetic properties can be calculated by Rytov's formulas [3]. However this condition is hardly achieved in the telecom band because it requires minimum feature sizes well below practically achievable limits. Thus, SWG devices are usually designed in the shallow subwavelength regime (i.e., just below the Bragg threshold), where Rytov's formulas do not hold and a careful modeling process is required for success. This process usually involves some preliminary steps in which the SWG waveguide behavior (refractive index, dispersion, anisotropy) is extracted from Bloch-Floquet analysis and then fed to subsequent design steps [4].

Several SWG based devices have been successfully developed in our group. Fiber-to-chip SWG based surface couplers which combine a diffractive grating in the propagation direction and a SWG grating in the transversal direction have been demonstrated which simultaneously attain good coupling efficiency and are simple to fabricate [5]. As typical grating based couplers lack from wideband performance, a new broadband zeroth diffraction order coupler has been proposed with an unprecedented efficiency-bandwidth product ( $0.4 \mathrm{~dB}$ efficiency and $126 \mathrm{~nm} 1 \mathrm{~dB}$ bandwidth) [6]. SWG metamaterials have also been used to design an ultrabroadband Multimode Interference (MMI) coupler with a potential 500nm bandwidth [7][8]. Measurement results for this device have experimentally shown greater than 300nm bandwidth with excellent performance (imbalance and phase error below $1 \mathrm{~dB}$ and $5^{\circ}$, respectively) which makes this device one of the most broadband silicon photonics device ever reported. Based on this device a wideband mode multiplexer has also been demonstrated [9]. Recently, we have realized that SWG birefringence can be controlled by tilting the SWG segments [4] and based in this a new polarization beam splitter has been developed [10]. 
Anisotropy modeling has also allowed to use GRIN lens fundamentals to design a spot size (x30) converter with subdecibel insertion losses and reduced footprint [11]. Using a different approach [12] an extreme spot size converter (x75) has been designed based on a sidewall-grating distributed Bragg deflector. This novel device allows a well-controlled coupling from a waveguide mode to a 2D beam freely propagating in the chip plane, open exciting prospects for freespace inspired optical beam manipulation in photonic chips. More recently, by using SWG waveguides loaded with lateral silicon segments [13] we have experimentally shown that it is possible to tightly control the grating strength allowing to design ultra-narrow band $(150 \mathrm{pm})$ Bragg filters [14]. In this contribution we will review some of the work that our group carried out in the last years to design high performance SWG based devices.

\section{EXTREME BEAM CONVERTER BASED ON BRAGG DEFLECTOR}

\subsection{Introduction to distributed Bragg deflectors}

Although channel waveguides, providing two dimensional light confinement, are by far the dominant guiding mechanism in PIC design, there are important devices, such as star couplers used in Arrayed Waveguide Gratings (AWGs), which are based in one dimensional confinement, i.e., in the use of beams that propagate freely in the chip plane while still vertically confined in a slab. Besides this relevant component, the possibilities of coupling from 2D confined waveguide modes to $1 \mathrm{D}$ vertically trapped slab beams is a widely unexplored territory.

Recently an optical antenna has been presented [15] capable of launching a 160x160 $\mu \mathrm{m} 2$ free space beam from a silicon wire $0.3 \times 0.25 \mu \mathrm{m} 2$ mode ( $\sim \mathrm{x} 105$ times in area) in an extremely compact footprint. The core of the device is a waveguide-to-slab beam coupler which is based in controlling the lateral leakage between a silicon wire and a slab situated in proximity.

Another type of waveguide-to-slab beam coupler, based on diffraction instead of leakage, was proposed by Stoll in 1978 [16] and the device was named distributed Bragg deflector (DBD). The same concept was used in [17] and in [18][19] where a DBD based in a smart combination of a sidewall grating and SWG material was successfully used to design, and experimentally demonstrate a wavelength demultiplexer in a silicon platform.

Our group has recently proposed an efficient procedure for designing DBD devices by means of Floquet-Bloch mode analysis and applied it to design a DBD-based beam expander [ref]. This procedure enables an efficient design independently each diffractive element that composes the DBD in order to achieve a targeted field profile inside a silicon slab.

\subsection{Distributed Bragg deflector design and verification}

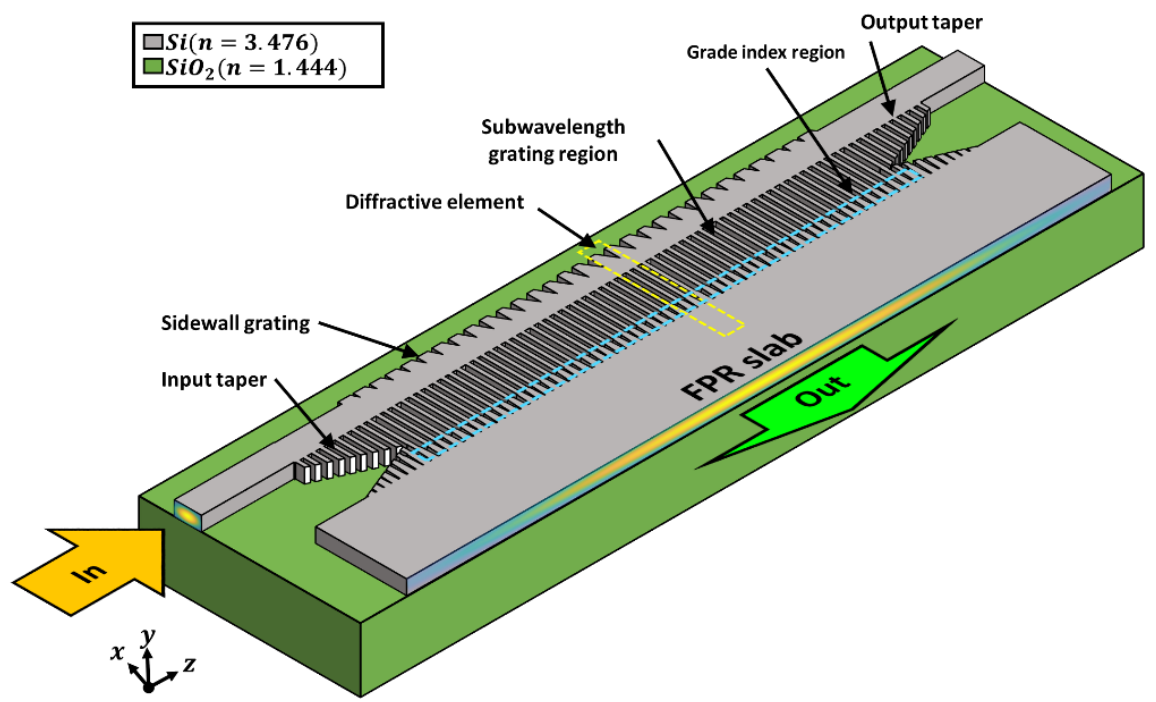

Figure 1. Geometry of the distributed Bragg deflector on SOI platform designed in [12]. 
A DBD device for the SOI platform was designed following the proposed procedure. The designed device, shown in figure 1, comprises a diffractive region, and two tapers that adiabatically connect the DBD region to standard silicon wire waveguides. The diffractive region is composed by a channel waveguide with a blazed sidewall, hence, the light propagating in along the channel waveguide is progressively diffracted to the lateral cladding slab made up of an SWG metamaterial. Finally, the light is transferred from the SWG slab to a silicon free propagation region (FPR) through a graded index region formed by triangular subwavelength structures as shown in figure 1.

FDTD simulation of the designed geometry demonstrated that $60 \%$ that enters the device is coupled to the FPR slab and field profile matches the designed one as shown in figure 2. The lost power has been mainly radiated out of the chip plane.

a)

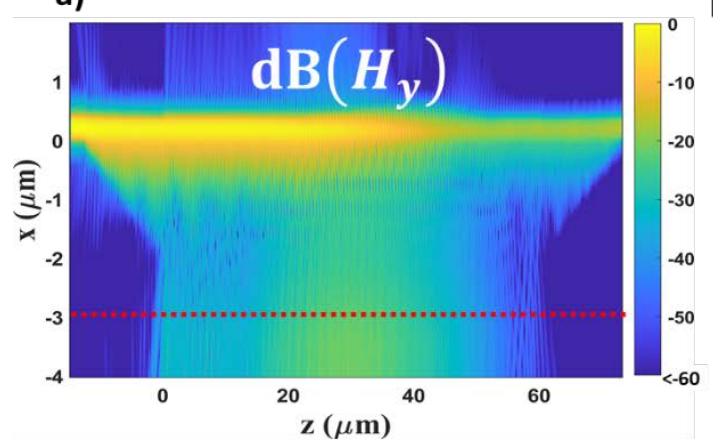

b)

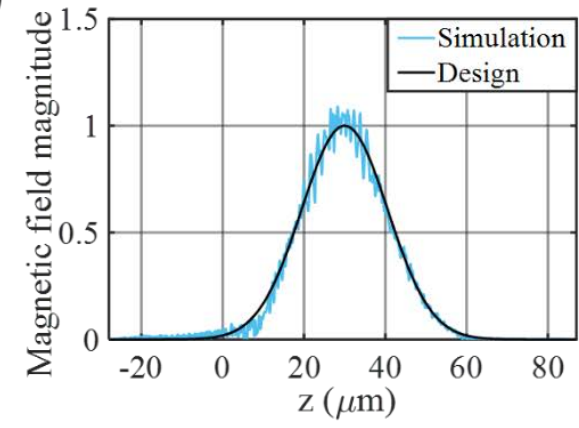

Figure 2. 3D FDTD simulation result for the DBD designed in [12]. Magnetic field amplitude at the middle of the silicon layer is shown in a). The field along the red dotted line is shown in b).

Preliminary experimental verification of a partial deflector was carried out. Figure 3 compares the experimental wavelength response with the simulated for the fabricated device. The measurement is in good agreement with the simulation, hence, demonstrating the validity of the design technique proposed.

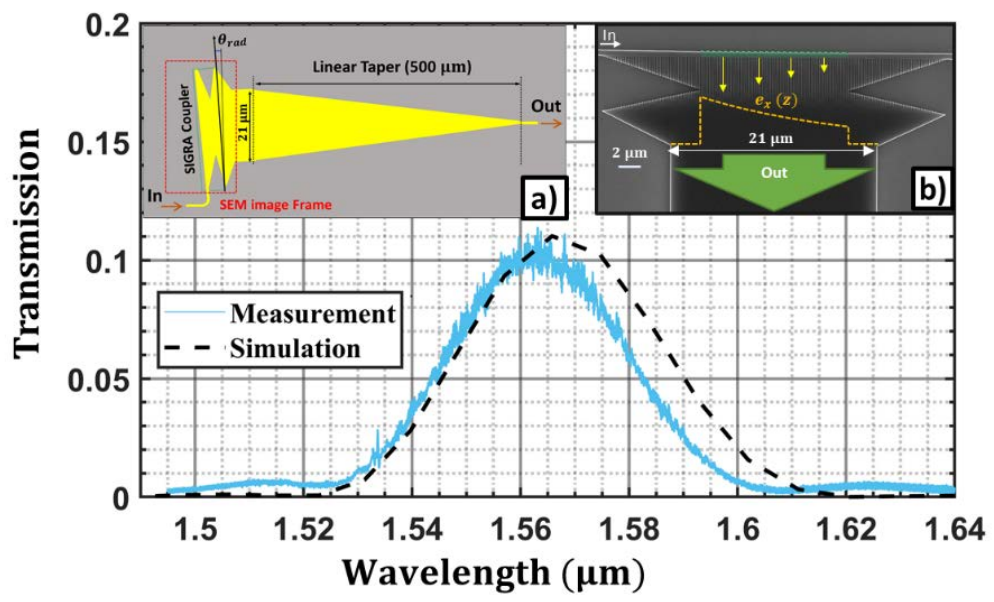

Figure 3.Wavelength response for the fabricated partial response and simulated response. Inset a) shows the schematic of the test structure and inset b) shows a SEM image of the fabricated device [12].

\section{ZERO-ORDER GRATING COUPLER}

One of the main challenges in silicon photonics is that of the fiber-chip coupling of light [20][21]. Because the crosssection sizes of optical waveguides and conventional single-mode optical fibers are significantly different, the modal mismatch prevents direct butt-coupling of light through the chip facet. To address this fundamental problem, surface grating couplers are commonly used. Surface grating couplers comprise a periodically or quasi-periodically perturbed 
waveguide that diffracts the incident power into an off-chip free-propagating plane wave. This radiated field can be collected by an optical fiber judiciously positioned above the chip with the right tilt angle. Unlike edge couplers, surface grating couplers offer attractive advantages, such as wafer-scale testing and robust misalignment tolerance, but typically have narrow bandwidth and low coupling efficiency [20]. Different approaches have been reported that circumvent these limitations, by broadening the bandwidth [22] or by increasing the coupling efficiency [23]. However, in most cases, there is a trade-off between both metrics, so that only one of them can be optimized [20][24]. In this invited talk, we review our design of a new surface grating coupler concept that, by radiating in the zeroth diffraction orders, overcomes the intrinsic bandwidth limitation of conventional grating couplers while optimizes the coupling efficiency [6].

Figure 4 shows a 3D schematic representation of a zero-order grating coupler. The bandwidth enhancement is achieved by (1) operating in the subwavelength regime, which frustrates all potential diffraction orders, and (2) assisting the grating with a high-refractive-index prism, which enables radiation in the zeroth order. The coupling efficiency is optimized (up to $\sim 95 \%$ ) by adjusting the tilt angle of the prism on top of the chip, according to the prism-film coupling theory [25].

Following this novel grating coupling concept, we designed a zero-order grating coupler at a wavelength of $1.55 \mu \mathrm{m}$ with a coupling efficiency of $-0.4 \mathrm{~dB}$ and a $1 \mathrm{~dB}$ bandwidth of $126 \mathrm{~nm}$, as shown in Fig. 4 [6]. This performance yields a coupling efficiency-bandwidth product of more than $100 \mathrm{~nm}$, which is substantially higher than the $60 \mathrm{~nm}$ of the best grating couplers yet reported in the literature [26].
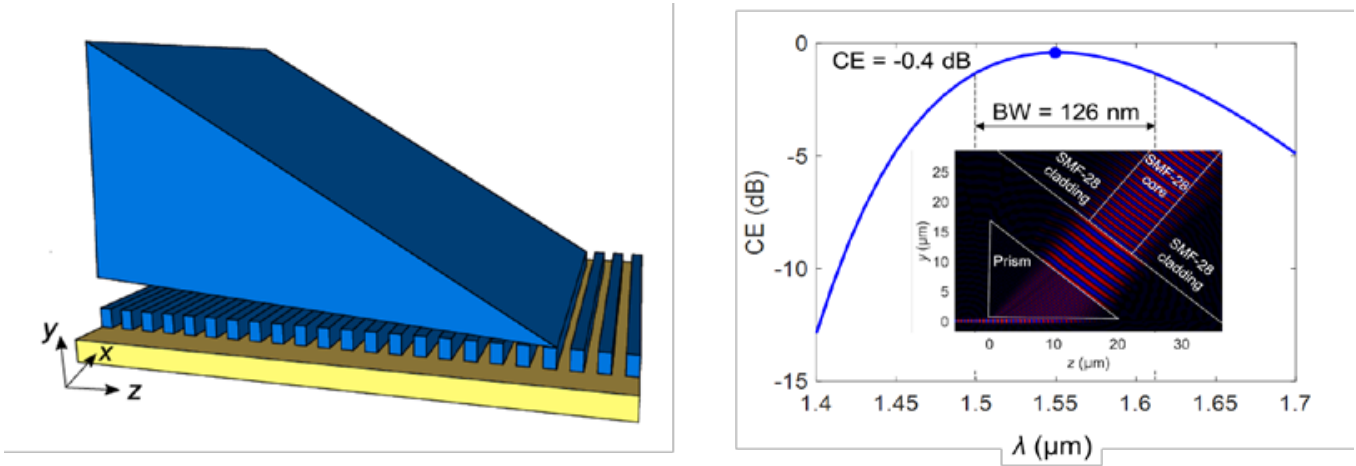

Figure 4. (Left) 3D schematic representation of a zero-order grating coupler, formed by a subwavelength grating waveguide that is assisted by a high-index, silicon prism to radiate in the zeroth diffraction order. (Right) Simulated coupling efficiency as a function of the wavelength. A peak coupling efficiency of $-0.4 \mathrm{~dB}$ and a broad bandwidth of $126 \mathrm{~nm}$ are theoretically achieved. In the inset, a 2D FDTD electric field propagation is included for the wavelength of $1.55 \mu \mathrm{m}$ and TE polarization.

\section{SWG INTEGRATED BRAGG FILTERS}

Although Bragg gratings have been mostly implemented in optical fibers, many efforts have also been devoted to developing Bragg gratings in planar waveguides. Typically, integrated Bragg gratings are designed by introducing a periodic variation on the waveguide width, which is also known as sidewall corrugation. In this case, the filter bandwidth, that is directly related to the grating strength $\kappa$, can be controlled by adjusting the amplitude of the corrugation. Nevertheless, narrowband spectral filters may require small corrugations difficult to be fabricated in practice, especially when using high-index contrast platforms such as silicon on insulator.

Recently, we have reported a new geometry of Bragg grating consisting of a SWG waveguide core and an array of silicon segments placed aside [13][14] (see Fig. 5a). These loading segments disturb evanescently the Floquet-Bloch mode propagating along the SWG core and comprise the Bragg grating itself. The SWG core is used to delocalize the electromagnetic field profile. This permits an accurate control of the grating strength through the separation $s$ between SWG core and loading segments, while keeping minimum feature sizes large enough for fabrication feasibility. Silicon Bragg filters with bandwidths ranging from $8 \mathrm{~nm}$ to $150 \mathrm{pm}$ have been demonstrated by using this geometry [14].

Our recent advances show that filters with an arbitrary spectral response can be designed by modulating along the grating length the separation between SWG core and loading segments, $s_{n}$, and the length of the Bragg periods, $\Lambda_{n}$ (see Fig $5 b$ ). 
As an example, in Fig. 1c it is represented the simulated response of a filter designed for the synthesis of 20 spectral notches at arbitrary wavelengths with a 3-dB bandwidth of $\sim 400 \mathrm{pm}$, which can be of interest in astronomical observation applications requiring the elimination of hydroxyl $(\mathrm{OH})$ emission lines from the Earth's atmosphere [27].

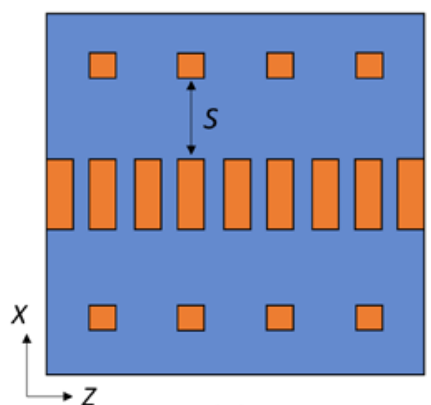

(a)

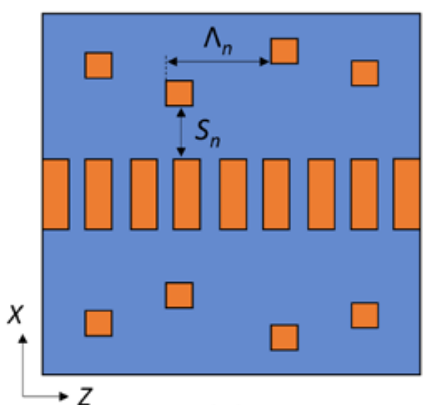

(b)

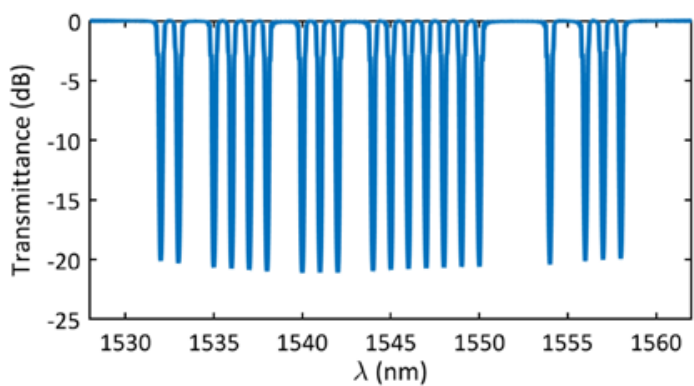

(c)

Figure 5. (a) Schematic view of the SWG Bragg filter geometry proposed in [13][14]. (b) Schematic view of the geometry proposed to implement filters with an arbitrary spectral response. (c) Simulated response of a filter designed for the elimination of 20 hydroxyl emission lines from the atmosphere in astronomical observation applications.

\section{ULTRA-BROADBAND PHASE SHIFTER}

Optical phase shifting is a primary operation in photonic integrated circuits targeting high-end applications such as communications [28], sensing [29] and quantum computing [30]. Most research efforts have been focused in the implementation of active phase shifters (PSs), which allow to dynamically tune the phase shift response between the involved signals [30]. On the contrary, passive PSs have barely evolved [31] and are usually replaced by active PSs to compensate for possible fabrication errors or to adjust the response for different wavelength. To overcome the bandwidth and fabrication constraints posed by state-of-the-art passive PSs based on waveguides of equal-length, we exploit the inherent anisotropy of SWG waveguides in combination with dispersion engineering. The proposed PS [shown in Fig. 6(a)] relies on two parallel SWG waveguides of dissimilar widths $\left(W_{A}\right.$ and $\left.W_{B}\right)$ but the same period $(\Lambda)$ and duty cycle $(a / \Lambda)$ [32]. SWG waveguides should be wide enough to hold paraxiality condition [33], whereas SWG tapers are incorporated to perform an adiabatic transition between the non-periodic interconnection waveguides of width $W_{I}$ and the corresponding SWG waveguide. As a prove of concept a $90^{\circ}$ PS was designed and our Floquet-Bloch simulation predicted a phase shift error below $1.7^{\circ}$ over an outstanding $400 \mathrm{~nm}$ bandwidth $(1.35-1.75 \mu \mathrm{m})$, maintaining the flat response even for fabrication errors of $\pm 20 \mathrm{~nm}$. The device was fabricated on the SOI platform and experimental results [depicted in Fig. 6(b)] showed a fourfold reduction in the phase variation compared to other conventional PS within the $145 \mathrm{~nm}$ wavelength range of our measurement setup.
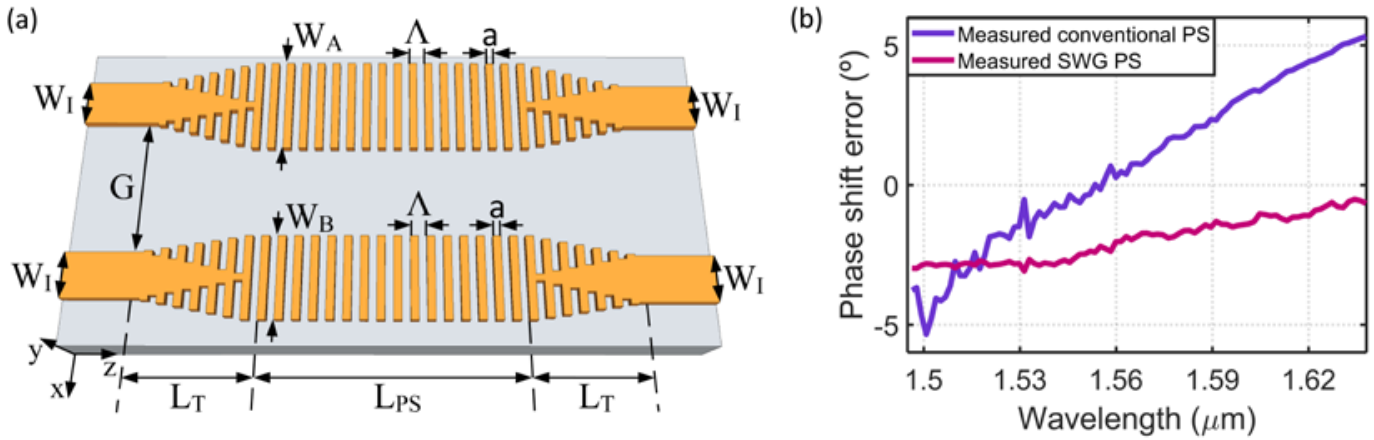

Figure 6. (a) Schematic of the proposed ultra-broadband PS. (b) Simulated and measured phase shift error of the designed $90^{\circ}$ phase shifter. Performance is compared to a state-of-the-art phase shifter based on back-to-back tapers [11]. 


\section{ACKNOWLEDGEMENTS}

The authors acknowledge funding from Alcyon Photonics (ref. $\mathrm{n}^{\circ}$ 8.06/5.59.5045), Universidad de Málaga, Ministerio de Economía y Competitividad (MINECO) (TEC2016-80718-R), Ministerio de Educación, Cultura y Deporte (MECD) (FPU16/06762, FPU17/00638, FPU16/03401), and Fondo Europeo de Desarrollo Regional—FEDER

\section{REFERENCES}

[1] Przemek J. Bock, Pavel Cheben, Jens H. Schmid, Jean Lapointe, André Delâge, Siegfried Janz, Geof C. Aers, Dan-Xia Xu, Adam Densmore, and Trevor J. Hall, "Subwavelength grating periodic structures in silicon-oninsulator: a new type of microphotonic waveguide," Opt. Express 18, 20251-20262 (2010)

[2] P. Cheben, R. Halir, J. H. Schmid, H.A. Atwater and D.R. Smith, "Subwavelength integrated photonics", Nature 560, 565 (2018).

[3] Halir, R., Ortega-Moñux, A., Benedikovic, D., Mashanovich, G. Z., Wangüemert-Pérez, J. G., Schmid, J. H., ... \& Cheben, P. (2018), "Subwavelength-grating metamaterial structures for silicon photonic devices”, Proceedings of the IEEE, 106(12), 2144-2157.

[4] José Manuel Luque-González, Alaine Herrero-Bermello, Alejandro Ortega-Moñux, Íñigo Molina-Fernández, Aitor V. Velasco, Pavel Cheben, Jens H. Schmid, Shurui Wang, and Robert Halir, "Tilted subwavelength gratings: controlling anisotropy in metamaterial nanophotonic waveguides," Opt. Lett. 43, 4691-4694 (2018)

[5] Halir, R., Cheben, P., Schmid, J. H., Ma, R., Bedard, D., Janz, S., ... \& Molina-Fernández, I. (2010), "Continuously apodized fiber-to-chip surface grating coupler with refractive index engineered subwavelength structure”, Optics letters, 35(19), 3243-3245

[6] A. Sánchez-Postigo et al., Broadband fiber-chip zero-order surface grating coupler with 0.4 dB efficiency, Optics Letters 41, 3013 (2016).

[7] A. Maese-Novo, R. Halir, S. Romero-García, D. Pérez-Galacho, L. Zavargo-Peche, A. Ortega-Moñux, I. Molina-Fernández, J. G. Wangüemert-Pérez, and P. Cheben, "Wavelength independent multimode interference coupler" Opt. Express 21, 7033-7040 (2013)

[8] Halir, R. , Cheben, P. , Luque-González, J. M., Sarmiento-Merenguel, J. D., Schmid, J. H., Wangüemert-Pérez, G. , Xu, D. , Wang, S. , Ortega-Moñux, A. and Molina-Fernández, Í., Ultra-broadband nanophotonic beamsplitter using an anisotropic sub-wavelength metamaterial. Laser \& Photonics Reviews, 10: 1039-1046, 2016. doi:10.1002/lpor.201600213

[9] D. González-Andrade et al., "Ultra-Broadband Mode Converter and Multiplexer Based on Sub-Wavelength Structures," in IEEE Photonics Journal, vol. 10, no. 2, pp. 1-10, April 2018, Art no. 2201010. doi: 10.1109/JPHOT.2018.2819364

[10] A. Herrero-Bermello, J. M. Luque-González, A. V. Velasco, A. Ortega-Moñux, P. Cheben and R. Halir, "Design of a Broadband Polarization Splitter Based on Anisotropy-Engineered Tilted Subwavelength Gratings," in IEEE Photonics Journal, vol. 11, no. 3, pp. 1-8, June 2019, Art no. 6601508. doi: 10.1109/JPHOT.2019.2912335

[11] Luque-González, J. M., Halir, R., Wangüemert-Pérez, J. G., de-Oliva-Rubio, J., Schmid, J. H., Cheben, P., ... \& Ortega-Moñux, A., “An Ultracompact GRIN-Lens-Based Spot Size Converter using Subwavelength Grating Metamaterials”, Laser \& Photonics Reviews 2019

[12] Hadij-ElHouati, A., Cheben, P., Ortega-Moñux, A., Wangüemert-Pérez, J. G., Halir, R., Schmid, J. H., \& Molina-Fernández, Í., “Distributed Bragg deflector coupler for on-chip shaping of optical beams”, Optics Express, 27(23), 33180-33193, 2019

[13] Čtyroký, J., Wangüemert-Pérez, J. G., Kwiecien, P., Richter, I., Litvik, J., Schmid, J. H., ... \& Cheben, P. (2018). Design of narrowband Bragg spectral filters in subwavelength grating metamaterial waveguides. Optics express, 26(1), 179-194.

[14]P. Cheben et al., Bragg filter bandwidth engineering in subwavelength grating metamaterial waveguides," Opt. Lett. 44, 1043 (2019). 
[15] S. Kim, D. A. Westly, B. J. Roxworthy, Q. Li, A. Yulaev, K. Srinivasan, and V. A. Aksyuk, "Photonic waveguide to free-space Gaussian beam extreme mode converter,” Light Sci. Appl., vol. 7, no. 1, p. 72, Dec. 2018.

[16]H. M. Stoll, “Distributed Bragg deflector: a multifunctional integrated optical device,” Appl. Opt., vol. 17, no. 16, pp. 2562-2569, Aug. 1978.

[17] R. L. Davis, W. Long, C.-J. Wang, T. Lam, J. G. Ho, P. M. Nachman, J. Poylio, O. V. Mishechkin, and M. Fallahi, "Distributed Bragg deflectors fabricated in sol-gel based waveguides,” IEEE Photonics Technol. Lett., vol. 16, no. 2, pp. 464-466, Feb. 2004.

[18] P. J. Bock, P. Cheben, A. Delâge, J. H. Schmid, D.-X. Xu, S. Janz, and T. J. Hall, "Demultiplexer with blazed waveguide sidewall grating and sub-wavelength grating structure,” Opt. Express, vol. 16, no. 22, pp. 1761617625, Oct. 2008.

[19] P. J. Bock, P. Cheben, J. H. Schmid, A. V Velasco, A. Delâge, S. Janz, D.-X. Xu, J. Lapointe, T. J. Hall, and M. L. Calvo, "Demonstration of a curved sidewall grating demultiplexer on silicon,” Opt. Express, vol. 20, no. 18, pp. 19882-19892, 2012.

[20] Taillaert, D., Bienstman, P. and Baets, R., “Compact efficient broadband grating coupler for silicon-on-insulator waveguides,” Optics Letters 23(29), 2749-2751 (2004).

[21] Marchetti, R., Lacava, C., Carroll, L., Gradkowski, K. and Minzioni, P., "Coupling strategies for silicon photonics integrated chips [Invited],” Photonics Research 7(2), 201-239 (2019).

[22]Zhong, Q., Veerasubramanian, V., Wang, Y., Shi, W., Patel, D., Ghosh, S., Samani, A., Chrostowski, L., Bojko, R. and Plant, D. V., "Focusing-curved subwavelength grating couplers for ultra-broadband silicon photonics optical interfaces,” Optics Express 22(15), 18224-18231 (2014).

[23] Hoppe, N., Sfar Zaoui, W., Rathgeber, L., Wang, Y., Klenk, R. H., Vogel, W., Kaschel, M., Portalupi, S. L., Burghartz, J. and Berroth, M., "Ultra-efficient silicon-on-insulator grating couplers with backside metal mirrors,” IEEE Journal of Selected Topics in Quantum Electronics 26(2), 8200206 (2020).

[24] Passoni, M., Gerace, D., Carroll, L. and Andreani, L. C., "Grating couplers in silicon on-insulator: The role of photonic guided resonances on lineshape and bandwidth,” Applied Physics Letters 110, 041107 (2017).

[25] Ulrich, R., “Optimum excitation of optical surface waves,” Journal of the Optical Society of America 61(11), 1467-1477 (1971).

[26] Sacher, W. D., Huang, Y., Ding, L., Taylor, B. J. F., Jayatilleka, H., Lo, G.-Q. and Poon, J. K. S., “Wide bandwidth and high coupling efficiency Si3N4-on-SOI dual-level grating coupler,” Optics Express 22(9), 10938-10947 (2014).

[27]T. Zhu, Y. Hu, P. Gatkine, S. Veilleux, J. Bland-Hawthorn, and M. Dagenais, "Arbitrary on-chip optical filter using complex waveguide Bragg gratings," Appl. Phys. Lett. 108, 101104 (2016).

[28] Doerr, C. R., Winzer, P. J., Chen, Y. K., Chandrasekhar, S., Rasras, M. S., Chen, L., Liow, T.-Y., Ang, K.-W. and Lo, G. Q., "Monolithic polarization and phase diversity coherent receiver in silicon," J. Lightwave Technol. 28(4), 520-525 (2010).

[29] Wangüemert-Pérez, J. G., Hadij-ElHouati, A., Sánchez-Postigo, A., Leuermann, J., Xu, D.-X., Cheben, P., Ortega-Moñux, A., Halir, R. and Molina-Fernández, Í., "Subwavelength structures for silicon photonics biosensing," Opt. Laser Technol. 109, 437-448 (2019).

[30] Harris, N. C., Bunandar, D., Pant, M., Steinbrecher, G. R., Mower, J., Prabhu, M., Baehr-Jones, T., Hochberg, M. and Englund, D., "Large-scale quantum photonic circuits in silicon," Nanophotonics 5(3), 456-468 (2016).

[31] Morrissey, P. E. and Peters, F. H. "Multimode interference couplers as compact and robust static optical phase shifters," Opt. Commun. 345, 1-5.

[32] González-Andrade, D., Luque-González, J. M., Wangüemert-Pérez, J. G., Ortega-Moñux, A., Cheben, P., Molina-Fernández, Í. and Velasco, A. V. (2019), "Ultra-broadband nanophotonic phase shifter based on subwavelength metamaterial waveguides," arXiv preprint arXiv:1907.07947 (2019).

[33] Soldano, L. B. and Pennings, E. C., "Optical multi-mode interference devices based on self-imaging: principles and applications," J. Lightwave Technol. 13(4), 615-627 (1995). 